\title{
Efficacy and safety of loxoprofen hydrogel patch versus loxoprofen tablet in patients with ankylosing spondylitis: A 4-week randomized, open-label study
}

\author{
MEIDA FAN ${ }^{1}$, SHUANGYAN CAO ${ }^{2}$, LIUDAN TU², QIUJING WEI ${ }^{2}$, \\ RIWEI YUAN ${ }^{2}$, XUEFENG $\mathrm{LI}^{3-5}$ and JIERUO GU ${ }^{2}$
}

\begin{abstract}
${ }^{1}$ Department of Rheumatology, Nanfang Hospital, Southern Medical University, Guangzhou, Guangdong 510515;
${ }^{2}$ Department of Rheumatology, The Third Affiliated Hospital of Sun Yat-Sen University, Guangzhou, Guangdong 510630;

${ }^{3}$ The Sixth Affiliated Hospital of Guangzhou Medical University, Qingyuan People's Hospital, Sino-French Hoffmann Institute,

School of Basic Medical Sciences, Guangzhou Medical University, Guangzhou, Guangdong 511436;

${ }^{4}$ Shenzhen Luohu People's Hospital, The Third Affiliated Hospital of Shenzhen University, Shenzhen, Guangdong 518001;

${ }^{5}$ Key Laboratory of Regenerative Biology, Guangdong Provincial Key Laboratory of Stem Cell and Regenerative

Medicine, South China Institute for Stem Cell Biology and Regenerative Medicine, Guangzhou Institutes of

Biomedicine and Health, Chinese Academy of Sciences, Guangzhou, Guangdong 510530, P.R. China
\end{abstract}

Received July 24, 2018; Accepted April 1, 2019

DOI: $10.3892 /$ br.2019.1209

\begin{abstract}
The aim of the present study was to assess the efficacy and safety of loxoprofen sodium hydrogel patch (LX-P) vs. loxoprofen sodium tablet (LX-T) in patients with active ankylosing spondylitis (AS). The study population consisted of patients who met the modified New York radiographic criteria for AS and had active disease. Patients were randomly assigned to either the LX-P group (LX-P $100 \mathrm{mg}$ per day) or LX-T group (LX-T $60 \mathrm{mg} 3$ times daily) for 4 weeks. The primary efficacy endpoint was the percentage of patients reaching Assessment in Ankylosing Spondylitis 20\% (ASAS20) response at week 4. Secondary efficacy outcomes included ASAS5/6 response rate and changes from baseline to week 4 for Ankylosing Spondylitis Disease Activity Score, patient's global assessment of disease activity, and pain score. Of the 70 randomized patients included, 35 patients were allocated to the LX-P group and 35 to the LX-T group. No significant differences were observed between the LX-P and LX-T groups in the proportion of patients achieving
\end{abstract}

Correspondence to: Dr Jieruo Gu, Department of Rheumatology, The Third Affiliated Hospital of Sun Yat-Sen University, 600 Tianhe Road, Tianhe, Guangzhou, Guangdong 510630, P.R. China

E-mail: gujieruo@163.com

Dr Xuefeng Li, The Sixth Affiliated Hospital of Guangzhou Medical University, Qingyuan People's Hospital, Sino-French Hoffmann Institute, School of Basic Medical Sciences, Guangzhou Medical University, Xinzao, Panyu, Guangzhou, Guangdong 511436, P.R. China

E-mail: xuefengli@gzhmu.edu.cn

Key words: ankylosing spondylitis, loxoprofen, hydrogel patch, topical application
ASAS20 response at week 4 (54.3 vs. $74.3 \%$; $\mathrm{P}=0.081)$, nor in the ASAS5/6 response and changes of efficacy outcomes between the two groups. Furthermore, patients without peripheral arthritis in the LX-P group were more likely to achieve ASAS20 response. There was a decreased incidence of gastrointestinal adverse events in the LX-P group, but this was not significant. There was no significant differences in efficacy and safety between topical LX-P and oral LX-T administration for patients with active AS.

\section{Introduction}

Ankylosing spondylitis (AS) is an inflammatory rheumatic disease characterized by inflammatory back pain, morning stiffness of the spine and enthesitis $(1,2)$. It predominantly affects the axial skeleton, although peripheral joints may also be affected. Disease onset usually begins in the second or third decade of life. With the progression of disease, it may lead to structural and functional impairments, work disability and even loss of self-sufficiency $(3,4)$.

Non-steroidal anti-inflammatory drugs (NSAIDs) are strongly recommended for patients with AS $(5,6)$. In addition, several studies have shown that long-term use of NSAIDs may delay radiographic progression in patients with AS $(7,8)$. Oral NSAIDs is the most common route of administration. However, the use of oral NSAIDs may lead to gastrointestinal, cardiovascular and renal adverse events (9-13). Loxoprofen sodium (LX), a non-selective NSAID, has a particularly high risk of gastrointestinal disorders. Therefore, topically applied NSAIDs have been developed to decrease the risk of gastrointestinal adverse events while providing good levels of pain relief for local acute and chronic painful conditions $(14,15)$. Loxoprofen in LX hydrogel patches (LX-P) can directly penetrate into the 
affected site and provide pain relief. Topical LX-P has been demonstrated to be non-inferior to oral LX tablet (LX-T) in efficacy and safety for patients with knee osteoarthritis in a randomized, double bind, controlled trial (16).

To the best of our knowledge, no randomized controlled trials of topical LX-P have been conducted in patients with AS to date. The aim of the present 4 week study was to assess the efficacy and safety of topical LX-P compared with oral LX-T in the treatment of patients with active AS in China.

\section{Patients and methods}

Patients. Patients aged 18-65 years fulfilling the 1984 modified AS New York criteria (17) were eligible for inclusion. Patients should present with active disease, defined by a Bath AS Disease Activity Index (BASDAI) (18) of $\geq 4$ on a $0-10$ $\mathrm{cm}$ visual analog scale (VAS) or an Ankylosing Spondylitis Disease Activity Score using the C-reactive protein level (ASDAS-CRP) $(19,20)$ of $\geq 1.3$. Other defined inclusion criteria were: i) NSAIDs washout period of at least 5 days prior to randomization; ii) disease-modifying antirheumatic drugs washout period of at least 4 weeks prior to randomization; iii) corticosteroids washout period of at least 4 weeks prior to randomization; and iv) biological agents washout period of at least 3 months prior to randomization. Sulfasalazine was permitted if the patient was taking a stable dose for 3 months prior to study entry. Patients with peripheral joint involvement were also included. Adequate contraception throughout the trial was required in women of childbearing age.

Patients were excluded if they had peptic ulcer, unstable cardiac diseases, abnormal hepatic or renal function, hematologic diseases, psychosis, or malignancy. Patients who were allergic to LX were also excluded.

Trial design. The trial was a randomized open-label study conducted in the Department of Rheumatology of The Third Affiliated Hospital of Sun Yat-Sen University, (Guangzhou, China). The protocol was approved by the Ethics Committee of the Third Affiliated Hospital of Sun Yat-Sen University in accordance with the Declaration of Helsinki principles and the guidelines for Good Clinical Practice (21). The study was registered on 10 January 2019 at www.ClinicalTrials.gov (identifier NCT03800797). Informed consents were obtained from all patients.

Clinical visits were performed at baseline (week 0), week 2 , and week 4 , or the time of discontinuing treatment by the same investigator. The demographic and disease characteristics were recorded at baseline. Every visit should record clinical and laboratory variables, evaluate efficacy outcomes and monitor adverse events.

Treatment. Patients were randomized 1:1 to either the LX-P group or the LX-T group for 4 weeks using a computergenerated schedule. Treatment assignment was open to the investigators and patients. Each patient qualifying for treatment was dispensed the corresponding drug. LX-P (Lead Chemical Co., Ltd.) contained $50 \mathrm{mg}$ LX per patch, while LX-T (Daiichi Sankyo Co., Ltd.) contained $60 \mathrm{mg}$ LX. Patients in the LX-P group applied LX-P $100 \mathrm{mg}$ onto the pain spot of vertebra once daily starting at night. Patients in the LX-T group took LX-T $60 \mathrm{mg} 3$ times daily following meals. The doses for LX-P and LX-T were selected according to a previous clinical trial involving LX-P in knee osteoarthritis (16). Dose adjustments were not allowed during the study period. Usually, an optimal effect of an NSAID is reached no later than after 1-2 weeks (11), so the treatment duration was 4 weeks.

At the screening visit, concomitant therapies with gastrointestinal protective drugs, including misoprostol or proton pump inhibitors, were stopped if there was no history of gastroduodenal ulcers.

Efficacy evaluation. The primary efficacy endpoint was the percentage of patients reaching Assessment in Ankylosing Spondylitis 20\% (ASAS20) response (22) at weeks 2 and 4 , which was defined as an improvement of $\geq 20 \%$ and net improvement of $\geq 1$ unit (0-10-cm VAS) from baseline in $\geq 3$ of the following 4 domains, and absence of worsening in any domain: Patient's global assessment of disease activity (PTGA), pain assessment, Bath Ankylosing Spondylitis Functional Index (BASFI) (23) and clinical inflammation, determined by 2 morning stiffness-associated scores on the BASDAI. The secondary efficacy endpoint was the percentage of patients achieving ASAS5/6 response at weeks 2 and 4, defined as 20\% improvement in 5 of the following 6 domains: PTGA, pain assessment, BASFI, inflammation, spinal mobility and CRP. Other efficacy measures included mean changes from baseline to week 4 for ASDAS-CRP, PTGA, total and nocturnal pain scores, clinical inflammation, BASDAI and BASFI.

Safety evaluation. Safety evaluations, including adverse events, physical examination and clinical laboratory tests, were monitored by the investigators throughout the study.

Statistical analysis. Statistical analysis was performed using SPSS 20.0 software (SPSS, Chicago, IL, USA). Baseline demographic and disease characteristic variables were summarized using descriptive statistics. All efficacy endpoint analyses were conducted in the intention-to-treat (ITT) population, which consisted of all randomized patients who took at least 1 dose of treatment. We used the last observation carried forward technique for patients who did not adhere to the study protocol.

Using the ITT population, the ASAS20 and ASAS5/6 response rates were analyzed by Pearson $\chi 2$ test. Patients were also stratified based on the presence or absence of peripheral arthritis to determine the effect of peripheral joint involvement on treatment response. Changes of efficacy endpoints from baseline to week 4 were analyzed using the Student's t-test or Wilcoxon-Mann-Whitney test depending on the Shapiro-Wilk normality test in the ITT population. The safety assessment were also conducted on all randomized patients. The incidence of adverse events was compared between the LX-P and LX-T groups using Fisher's exact test. For all analyses, statistical analysis was performed at $\alpha=0.05$. $\mathrm{P}<0.05$ was considered to indicate a statistically significant difference.

\section{Results}

Enrollment and characteristics of patients. From May 2015 to December 2015, 82 patients were screened, and 70 eligible 


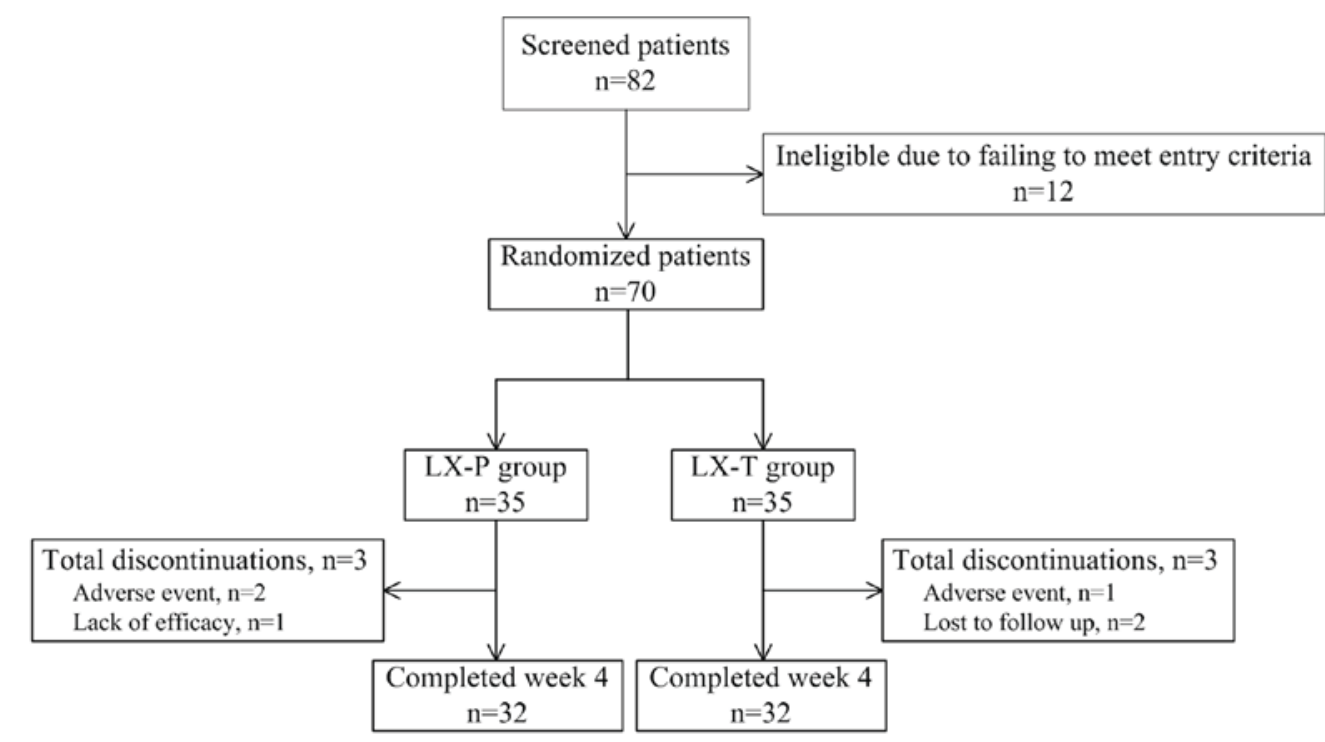

Figure 1. Flow diagram of the study design. LX-P, loxoprofen sodium hydrogel patch; LX-T, loxoprofen sodium tablet.

patients were randomized into the 4-week trial, with 35 in the LX-P group and 35 in the LX-T group (Fig. 1). In total, 6 of 70 patients withdrew from the study following randomization, 3 from the LX-P group and 3 from the LX-T group, resulting in an overall dropout rate of $8.6 \%$. The reasons for dropping out included adverse events, insufficient efficacy and loss to follow up.

At baseline, $48(68.6 \%)$ patients were male, and $22(31.4 \%)$ had peripheral arthritis. The baseline demographic and disease characteristics were similar between LX-P and LX-T groups (Table I).

Efficacy. The primary efficacy endpoints of ASAS20 response at weeks 2 and 4 in the ITT population are shown in Fig. 2A. No significant differences between the LX-P and LX-T groups in the proportion of patients achieving ASAS20 response at week 2 [13/35 (37.1\%) for LX-P group vs. 17/35 (48.6\%) for LX-T group; $\mathrm{P}=0.334]$ and week 4 [19/35 (54.3\%) for LX-P group vs. 26/35 (74.3\%) for LX-T group; P=0.081] were observed. In addition, no statistically significant differences for ASAS5/6 response at weeks 2 and 4 between the two groups were observed [week 2, 7/35 (20.0\%) vs. 9/35 (25.7\%); $\mathrm{P}=0.569$; week 4, 13/35 (37.1\%) vs. 16/35 (45.7\%); $\mathrm{P}=0.467$; Fig. 2B]. Changes in efficacy endpoints, including ASDAS-CRP, PTGA, total and nocturnal pain score, clinical inflammation, BASDAI and BASFI, from baseline to week 4 were compared between the LX-P and LX-T groups. There were also no significant differences between the two groups observed (all P>0.05; Table II).

In a subgroup analysis of patients without peripheral arthritis, an increase in the proportion of patients achieving ASAS20 response was observed in the LX-P group (Fig. 3). This suggested that patients without peripheral arthritis in the LX-P group were more likely to achieve ASAS20 response compared with those with peripheral joints involvement.

Safety. The incidence of any adverse events described during the study was similar $(\mathrm{P}=0.710)$ between the LX-P and LX-T groups: $3 / 35(8.6 \%)$ patients in the LX-P group and 5/35 $(14.3 \%)$ in the LX-T group (Table III). All adverse events were mild to moderate in intensity; no serious adverse events or mortalities were observed in either group. The most frequent adverse events for patients receiving LX-P were skin irritation or rashes, while the most common adverse event for patients receiving LX-T was gastrointestinal disorders.

\section{Discussion}

To date, only a few studies of osteoarthritis have compared the efficacy of topical vs. oral administration routes of NSAIDs $(16,24,25)$. To the best of our knowledge, there are no studies comparing topical vs. oral administration routes of NSAIDs in AS. The results of the present randomized controlled trial demonstrated that there were no significant differences in efficacy between topical LX-P and oral LX-T administration in patients with active AS, which is consistent with the results from a recent study in patients with knee osteoarthritis (16). In addition, topical LX-P was associated with non-significantly fewer gastrointestinal adverse events compared with oral LX-T.

In the present study, $31.4 \%$ of the patients had peripheral arthritis. However, LX-P treatments were only applied to the pain area of the vertebra, in order to maintain the same dosage. Considering that peripheral arthritis may affect the treatment response of topical LX-P, an exploratory analysis of patient subgroups with and without peripheral arthritis was performed. The results suggested that patients without peripheral arthritis in the LX-P group were more likely to achieve ASAS20 response. Therefore, we hypothesize that LX-P may have an improved efficacy in patients whose pain is localized in one area. However, additional investigation is required to explore the efficacy of topical LX-P in patients with AS, in particular those with generalized pain.

NSAIDs are recommended as the first-line drug option for the treatment of AS $(5,6)$, and long-term use of NSAIDs may delay the radiographic progression for patients with AS $(7,8)$. However, long-term exposure of NSAIDs may cause 
Table I. Baseline demographic and disease characteristics of the patients with ankylosing spondylitis.

\begin{tabular}{|c|c|c|c|}
\hline \multirow[b]{2}{*}{ Characteristics } & \multicolumn{2}{|c|}{ Groups } & \multirow[b]{2}{*}{ P-value } \\
\hline & LX-P $(n=35)$ & LX-T $(n=35)$ & \\
\hline \multicolumn{4}{|l|}{ Demographic characteristics } \\
\hline Age, years & $28(24-36)$ & $30(23-36)$ & 0.642 \\
\hline Male, no. $(\%)$ & $22(62.9)$ & $26(74.3)$ & 0.303 \\
\hline Height, cm & $165.3 \pm 7.9$ & $166.3 \pm 6.5$ & 0.575 \\
\hline Weight, kg & $58.5 \pm 11.6$ & $60.6 \pm 12.0$ & 0.465 \\
\hline Smoking, no. (\%) & $9(25.7)$ & $12(34.3)$ & 0.434 \\
\hline \multicolumn{4}{|l|}{ Disease characteristics } \\
\hline HLA-B27 positive, no. (\%) & $35(100.0)$ & $34(97.1)$ & 1.000 \\
\hline Disease duration, years & $5.0(2.0-9.0)$ & $4.0(1.0-9.0)$ & 0.773 \\
\hline Peripheral arthritis, no. (\%) & $9(25.7)$ & $13(37.1)$ & 0.303 \\
\hline \multicolumn{4}{|l|}{ Disease activity } \\
\hline PTGA, 0-10-cm VAS & $5.0(4.0-7.0)$ & $6.0(5.0-7.0)$ & 0.273 \\
\hline Total pain score, $0-10-\mathrm{cm}$ VAS & $5.0(3.0-7.0)$ & $5.0(4.0-7.0)$ & 0.472 \\
\hline Nocturnal pain score, $0-10-\mathrm{cm}$ VAS & $6.0(3.0-7.0)$ & $5.0(3.0-7.0)$ & 0.387 \\
\hline Clinical inflammation, 0-10-cm VAS & $3.8(2.8-6.5)$ & $3.8(2.8-5.0)$ & 0.557 \\
\hline BASDAI, 0-10-cm VAS & $4.3 \pm 1.4$ & $4.2 \pm 1.4$ & 0.735 \\
\hline BASFI, 0-10-cm VAS & $1.0(0.2-1.8)$ & $0.7(0.2-1.9)$ & 0.986 \\
\hline BASMI, 0-10-cm VAS & $1.0(0-2.0)$ & $1.0(0-3.0)$ & 0.514 \\
\hline $\mathrm{CRP}, \mathrm{mg} / \mathrm{l}$ & $5.9(1.9-16.8)$ & $8.5(5.3-17.6)$ & 0.109 \\
\hline ASDAS-CRP & $2.8 \pm 0.9$ & $3.0 \pm 0.7$ & 0.223 \\
\hline \multicolumn{4}{|l|}{ Prior medications } \\
\hline NSAIDs, no. (\%) & $30(85.7)$ & $29(82.9)$ & 0.743 \\
\hline DMARDs, no. (\%) & $12(34.3)$ & 8 (22.9) & 0.290 \\
\hline Biological agents, no. (\%) & $3(8.6)$ & $4(11.4)$ & 1.000 \\
\hline
\end{tabular}

Analyses were performed on the intention-to-treat population. Data are presented as the mean \pm standard deviation for height, weight, BASDAI, and ASDAS-CRP, while data concerning age, disease duration, PTGA, total and nocturnal pain score, clinical inflammation, BASFI, BASMI, and CRP are presented as the median and interquartile range. PTGA, patient's global assessment of disease activity; VAS, visual analog scale; BASDAI, Bath Ankylosing Spondylitis Disease Activity Index; BASFI, Bath Ankylosing Spondylitis Functional Index; BASMI, Bath Ankylosing Spondylitis Metrology Index; ASDAS-CRP, Ankylosing Spondylitis Disease Activity Score using the C-reactive protein level; NSAIDs, non-steroidal anti-inflammatory drugs; DMARDs, disease-modifying antirheumatic drugs.
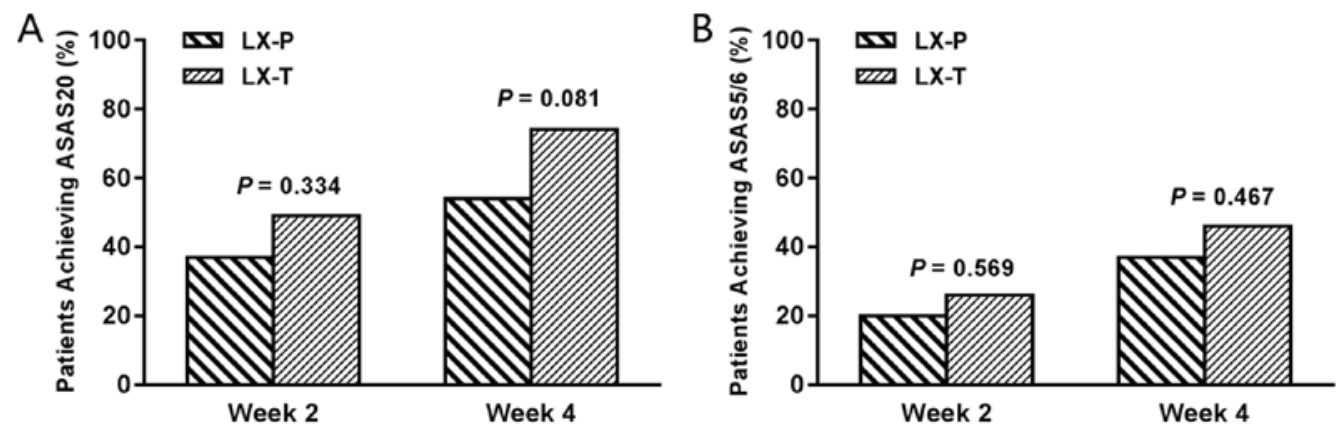

Figure 2. Primary and secondary endpoints. Proportion of patients achieving (A) ASAS20 and (B) ASAS5/6 responses at weeks 2 and 4. ASAS, Assessment in Ankylosing Spondylitis; LX-P, loxoprofen sodium hydrogel patch; LX-T, loxoprofen sodium tablet.

gastrointestinal, cardiovascular, and renal adverse events (9-13). Topical NSAIDs are considered to be associated with fewer gastrointestinal adverse events $(24,25)$. Topical LX-P allows LX to penetrate into the affected site directly instead of through the gastrointestinal tract. In the present study, there were no significant differences in safety between the LX-P and LX-T groups, probably due to the limited number of patients. Notably, skin disorders were the most frequently reported 
Table II. Changes in efficacy outcomes from baseline to week 4 in the LX-P and LX-T groups.

\begin{tabular}{lccr}
\hline & \multicolumn{2}{c}{ Groups } \\
\cline { 2 - 3 } Efficacy outcomes & LX-P $(\mathrm{n}=35)$ & LX-T $(\mathrm{n}=35)$ & P-value \\
\hline ASDAS-CRP & $-0.6 \pm 0.7$ & $-0.8 \pm 0.6$ & 0.301 \\
PTGA, 0-10-cm VAS & $-1.0(-3.0-0)$ & $-2.0(-2.0-0)$ & 0.724 \\
Total pain score, 0-10-cm VAS & $-1.0(-2.0-0)$ & $-2.0(-3.0--1.0)$ & 0.082 \\
Nocturnal pain score, 0-10-cm VAS & $-2.0(-3.0-0)$ & $-1.0(-3.0-0)$ & 0.214 \\
Clinical inflammation, 0-10-cm VAS & $-1.5(-2.8-0)$ & $-1.5(-3.0--0.5)$ & 0.415 \\
BASDAI, 0-10-cm VAS & $-1.5 \pm 1.2$ & $-1.7 \pm 1.2$ & 0.442 \\
BASFI, 0-10-cm VAS & $-0.1(-0.7-0)$ & $-0.2(-1.0-0)$ & 0.435 \\
\hline
\end{tabular}

Analyses were performed on the intention-to-treat population. Data are presented as the mean \pm standard deviation for ASDAS-CRP and BASDAI, and all other values are presented as the median and interquartile range. ASDAS-CRP, Ankylosing Spondylitis Disease Activity Score using the C-reactive protein level; PTGA, patient's global assessment of disease activity; VAS, visual analog scale; BASDAI, Bath Ankylosing Spondylitis Disease Activity Index; BASFI, Bath Ankylosing Spondylitis Functional Index.

Table III. Adverse events occurring in each group during the study.

\begin{tabular}{lcc}
\hline Event categories & $\begin{array}{c}\text { LX-P } \\
(\mathrm{n}=35)(\%)\end{array}$ & $\begin{array}{c}\text { LX-T } \\
(\mathrm{n}=35)(\%)\end{array}$ \\
\hline Any adverse event & $3(8.6)$ & $5(14.3)$ \\
Nausea & $0(0.0)$ & $1(2.9)$ \\
Dyspepsia & $0(0.0)$ & $1(2.9)$ \\
Skin pruritus & $2(5.7)$ & $1(2.9)$ \\
Dizziness & $0(0.0)$ & $1(2.9)$ \\
Upper respiratory tract infection & $1(2.9)$ & $1(2.9)$ \\
Serious adverse event & $0(0.0)$ & $0(0.0)$ \\
Adverse event leading & $2(5.7)$ & $1(2.9)$ \\
to discontinuation & & \\
\hline
\end{tabular}

Analyses were performed on the intention-to-treat population. Data are presented as the number $(\%)$ of patients.

treatment-associated adverse events in the LX-P group, but this was not significant. Furthermore, a decreased incidence of gastrointestinal disorders was observed in patients receiving LX-P. These results suggest that LX-P may be a potentially useful therapeutic drug with the advantages of easy administration and few gastrointestinal adverse events.

There are several limitations in the present study. Firstly, the study was a single-center, open-label trial, which may have introduced a certain level of bias into the analysis. Secondly, the sample size of the study may have been too small to draw definite conclusions. Therefore, to strengthen the results, several disease activity indexes were assessed. Thirdly, patients with peripheral joint involvement were enrolled. This may have affected the efficacy of LX-P. The subgroup analysis revealed that patients without peripheral arthritis in the LX-P group were more likely to achieve ASAS20 response. Fourthly, only the short-term efficacy and safety of topical

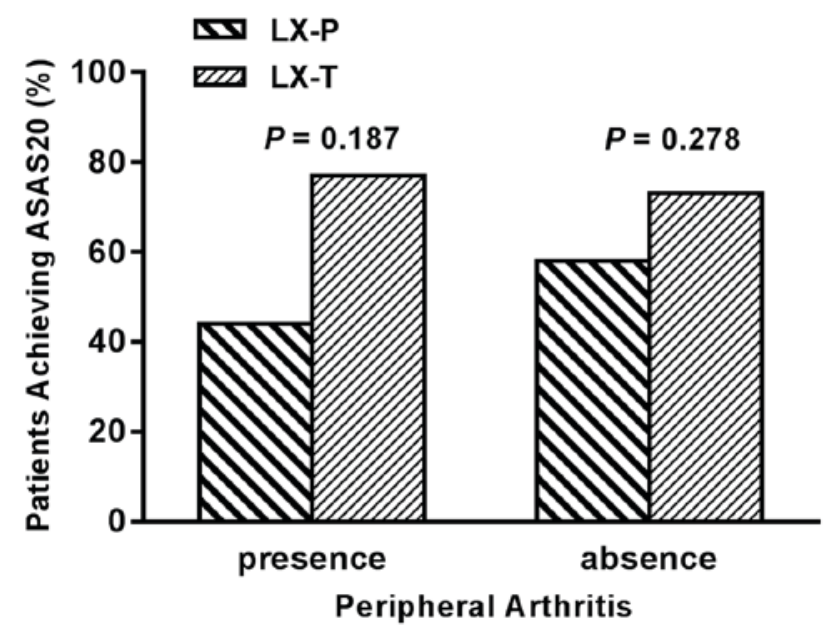

Figure 3. Proportion of patients achieving ASAS20 response at week 4 among those with or without peripheral arthritis at baseline. ASAS, Assessment in Ankylosing Spondylitis; LX-P, loxoprofen sodium hydrogel patch; LX-T, loxoprofen sodium tablet.

LX-P in the treatment of AS was investigated. Future studies with adequate follow-up are required to assess the long-term efficacy and safety of LX-P in AS.

In summary, the results of the present study suggest that there were no significant differences in efficacy between topical LX-P and oral LX-T for patients with active AS. In addition, topical LX-P was well tolerated and associated with few gastrointestinal adverse events.

\section{Acknowledgements}

Not applicable.

\section{Funding}

The present study was supported by National Natural Science Foundation of China (grant nos. 81571595 and 81702327), the President foundation of Nanfang Hospital, Southern Medical 
University (grant no. 2016C010), China Postdoctoral Science Foundation: 2018M640834, and Innovation Program of Shenzhen (grant no. JCYJ20180508165208399).

\section{Availability of data and materials}

The datasets analyzed during the current study are available from the corresponding author on reasonable request.

\section{Authors' contributions}

MF participated in the design of the study, clinical visits, data collection, design of the statistical analysis plan and wrote the first draft of the manuscript. SC and LT participated in clinical visits and critically reviewed the manuscript. QW and RY participated in data collection and critically reviewed the manuscript. JG conceived the study and participated in the interpretation of data. XL critically reviewed the manuscript and provided final approval of the manuscript.

\section{Ethics approval and consent to participate}

The present study was approved by the Third Affiliated Hospital of Sun Yat-Sen University, (Guangzhou, China); all patients provided written informed consent.

\section{Competing interests}

The authors declare that they have no competing interests.

\section{References}

1. Sieper J and Poddubnyy D: Axial spondyloarthritis. Lancet 390: 73-84, 2017.

2. Braun J and Sieper J: Ankylosing spondylitis. Lancet 369: 1379-1390, 2007.

3. Zink A, Braun J, Listing J and Wollenhaupt J: Disability and handicap in rheumatoid arthritis and ankylosing spondylitis - results from the German rheumatological database. German Collaborative Arthritis Centers. J Rheumatol 27: 613-622, 2000

4. Boonen A, Chorus A, Miedema H, van der Heijde D, van der Tempel $\mathrm{H}$ and van der Linden S: Employment, work disability, and work days lost in patients with ankylosing spondylitis: a cross sectional study of Dutch patients. Ann Rheum Dis 60: 353-358, 2001.

5. Ward MM, Deodhar A, Akl EA, Lui A, Ermann J, Gensler LS, Smith JA, Borenstein D, Hiratzka J, Weiss PF, Inman RD, et al: American College of Rheumatology/Spondylitis Association of America/Spondyloarthritis Research and Treatment Network 2015 Recommendations for the Treatment of Ankylosing Spondylitis and Nonradiographic Axial Spondyloarthritis. Arthritis Care Res (Hoboken) 68: 151-166, 2016.

6. van der Heijde D, Ramiro S, Landewe R, Baraliakos X, Van den Bosch F, Sepriano A, Regel A, Ciurea A, Dagfinrud H, Dougados M, et al: 2016 update of the ASAS-EULAR management recommendations for axial spondyloarthritis. Ann Rheum Dis 76: 978-991, 2017.

7. Wanders A, Heijde Dv, Landewé R, Béhier JM, Calin A, Olivieri I, Zeidler H and Dougados M: Nonsteroidal antiinflammatory drugs reduce radiographic progression in patients with ankylosing spondylitis: a randomized clinical trial. Arthritis Rheum 52: 1756-1765, 2005.
8. Poddubnyy D, Rudwaleit M, Haibel H, Listing J, Märker-Hermann E, Zeidler H, Braun J and Sieper J: Effect of non-steroidal anti-inflammatory drugs on radiographic spinal progression in patients with axial spondyloarthritis: results from the German Spondyloarthritis Inception Cohort. Ann Rheum Dis 71: 1616-1622, 2012.

9. Singh G and Triadafilopoulos G: Epidemiology of NSAID induced gastrointestinal complications. J Rheumatol Suppl 56: 18-24, 1999.

10. Fosbøl EL, Køber L, Torp-Pedersen C and Gislason GH: Cardiovascular safety of non-steroidal anti-inflammatory drugs among healthy individuals. Expert Opin Drug Saf 9: 893-903, 2010.

11. Song IH, Poddubnyy DA, Rudwaleit M and Sieper J: Benefits and risks of ankylosing spondylitis treatment with nonsteroidal antiinflammatory drugs. Arthritis Rheum 58: 929-938, 2008.

12. Shau WY, Chen HC, Chen ST, Chou HW, Chang CH, Kuo CW and Lai MS: Risk of new acute myocardial infarction hospitalization associated with use of oral and parenteral non-steroidal anti-inflammation drugs (NSAIDs): a case-crossover study of Taiwan's National Health Insurance claims database and review of current evidence. BMC Cardiovasc Disord 12: 4, 2012.

13. Shukla A, Rai MK, Prasad N and Agarwal V: Short-Term Non-Steroid Anti-Inflammatory Drug Use in Spondyloarthritis Patients Induces Subclinical Acute Kidney Injury: Biomarkers Study. Nephron 135: 277-286, 2017.

14. Massey T, Derry S, Moore RA and McQuay HJ: Topical NSAIDs for acute pain in adults. Cochrane Database Syst Rev 6: CD007402, 2010.

15. Derry S, Moore RA and Rabbie R: Topical NSAIDs for chronic musculoskeletal pain in adults. Cochrane Database Syst Rev 9: CD007400, 2012.

16. Mu R, Bao CD, Chen ZW, Zheng Y, Wang GC, Zhao DB, Hu SX, Li YJ, Shao ZW, Zhang ZY, et al: Efficacy and safety of loxoprofen hydrogel patch versus loxoprofen tablet in patients with knee osteoarthritis: a randomized controlled non-inferiority trial. Clin Rheumatol 35: 165-173, 2016

17. van der Linden S, Valkenburg HA and Cats A: Evaluation of diagnostic criteria for ankylosing spondylitis. A proposal for modification of the New York criteria. Arthritis Rheum 27: 361-368, 1984.

18. Garrett S, Jenkinson T, Kennedy LG, Whitelock H, Gaisford P and Calin A: A new approach to defining disease status in ankylosing spondylitis: the Bath Ankylosing Spondylitis Disease Activity Index. J Rheumatol 21: 2286-2291, 1994.

19. van der Heijde D, Lie E, Kvien TK, Sieper J, Van den Bosch F, Listing J, Braun J and Landewé R; Assessment of SpondyloArthritis international Society (ASAS): ASDAS, a highly discriminatory ASAS-endorsed disease activity score in patients with ankylosing spondylitis. Ann Rheum Dis 68: 1811-1818, 2009.

20. Machado P, Landewé R, Lie E, Kvien TK, Braun J, Baker D and van der Heijde D; Assessment of SpondyloArthritis international Society: Ankylosing Spondylitis Disease Activity Score (ASDAS): defining cut-off values for disease activity states and improvement scores. Ann Rheum Dis 70: 47-53, 2011.

21. Grimes DA, Hubacher D, Nanda K, Schulz KF, Moher D and Altman DG: The Good Clinical Practice guideline: a bronze standard for clinical research. Lancet 366: 172-174, 2005.

22. Anderson JJ, Baron G, van der Heijde D, Felson DT and Dougados M: Ankylosing spondylitis assessment group preliminary definition of short-term improvement in ankylosing spondylitis. Arthritis Rheum 44: 1876-1886, 2001.

23. Calin A, Garrett S, Whitelock H, Kennedy LG, O'Hea J, Mallorie P and Jenkinson T: A new approach to defining functional ability in ankylosing spondylitis: the development of the Bath Ankylosing Spondylitis Functional Index. J Rheumatol 21: 2281-2285, 1994.

24. Banning M: The use of topical diclofenac for pain in osteoarthritis of the knee: a review. Br J Community Nurs 11: 487-492, 2006.

25. Tugwell PS, Wells GA and Shainhouse JZ: Equivalence study of a topical diclofenac solution (pennsaid) compared with oral diclofenac in symptomatic treatment of osteoarthritis of the knee: a randomized controlled trial. J Rheumatol 31: 2002-2012, 2004. 